\section{Buchrezension zu: Chlamydia Biology}

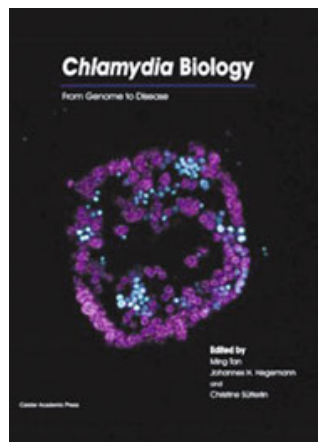

Chlamydia Biology

From Genome to Disease

Ming Tan, Johannes $\mathrm{H}$.

Hegemann und Christine

Sütterlin (Hrsg.)

482 S., Caister Academic Press, 2020

SC, £ 199,00.

ISBN: 9781912530281

Auch als E-Book erhältlich

DOI: $10.1007 / \mathrm{s} 12268-020-1405-3$

(C) Die Autorin 2020

Chlamydien sind perfekte $\mathrm{Pa}-$ rasiten. Sie machen es sich in ihrer intrazellulären Nische in Epithelzellen verschiedener Schleimhäute bequem, unbehelligt vom Immun-Radar ihrer Wirte. Nur ab und zu stören (unzureichend verstandene) Prozesse den trügerischen Frieden. Es kommt zu Entzündungsreaktionen mit entsprechenden Krankheitsbildern. Infektionen mit Chlamydia trachomatis zählen heute zu den weltweit am weitesten verbreiteten $\mathrm{Ge}$ schlechtskrankheiten und sind ein häufiger Grund für weibliche Unfruchtbarkeit. Dementsprechend groß ist das Interesse der klinischen und Grundlagenforschung an diesen kleinen Erregern.

Schon acht Jahre nach Erscheinen des letzten großen Reviewbandes (Chlamydiales, ASM Press) fanden die drei Herausgeber genug Neues und Berichtenswertes, um einen aktuellen Band im britischen Verlag Caister Academic Press vorzulegen. Wieder reicht das sorgfältig ausgewählte Spektrum der Themen von der Klinik der chlamydialen Human-, aber auch Tierinfektionen über die Mechanismen der intrazellulären Etablierung der Chlamydien und der Immunabwehr der Wirtszellen bis hin zu praktisch orientierten Kapiteln über Tiermodelle und Impfstoffentwicklung. Die rasanteste Entwicklung mit Auswirkungen auf die gesamte Chlamydienforschung gab es in den letzten Jahren auf den Gebieten Genetics (Etablierung von Transformationsmethoden) und Genomics (NGS und Sequenzanalyse), die jeweils mit einem Kapitel vertreten sind. Für begrüßenswert halte ich die Behandlung neuer, interessanter Themen wie chlamydiale Zellteilung sowie Chlamydien und Mikrobiota, für entbehrlich das Kapitel über den umstrittenen Virulenzfaktor CPAF.

Das neue Konzept der Herausgeber, jedes Kapitel von Autorenteams aus unterschiedlichen Forschungseinrichtungen mit individuellen Sichtweisen bearbeiten zu lassen, ist sehr gut aufgegangen. So geben die meisten Kapitel einen vielseitigen, gut und aktuell recherchierten, aber auch in sich kohärenten Einblick in ein Themengebiet, der zum Weiterlesen in der Originalliteratur anregt und befähigt. Das Buch als Ganzes sei Forschungsstudierenden und Doktorand/inn/en als Einstiegshilfe in die Welt der Chlamydiologie und Wissenschaftler/inne/n als aktuelle Orientierungshilfe empfohlen.

Christiane Schnee,

Friedrich-Loeffler-Institut Jena, Christiane.Schnee@fli.de

* Funding Open Access funding provided by Project DEAL.

* Open Access: Dieser Artikel wird unter der Creative Commons Namensnennung 4.0 International Lizenz veröffentlicht, welche die Nutzung, Vervielfältigung, Bearbeitung, Verbreitung und Wiedergabe in jeglichem Medium und Format erlaubt, sofern Sie den/die ursprünglichen Autor(en) und die Quelle ordnungsgemäß nennen, einen Link zur Creative Commons Lizenz beifügen und angeben, ob Ânderungen vorgenommen wurden. Die in diesem Artikel enthal- tenen Bilder und sonstiges Drittmaterial unterliegen ebenfalls der genannten Creative Commons Lizenz, sofern sich aus der Abbildungslegende nichts anderes ergibt. Sofern das betreffende Material nicht unter der genannten Creative Commons Lizenz steht und die betreffende Handlung nicht nach gesetzlichen Vorschriften erlaubt ist, ist für die oben aufgeführten Weiterverwendungen des Materials die Einwilligung des jeweiligen Rechteinhabers einzuholen. Weitere Details zur Lizenz entnehmen Sie bitte der Lizenzinformation auf http://creativecommons.org/ licenses/by/4.0/deed.de. 